\title{
In Vitro Biological Evaluation of 3-D Hydroxyapatite/Collagen (50/50 wt. (\%)) Scaffolds
}

\author{
Doris Moura Campos $^{a, b}$, Karine Anselme ${ }^{b}$, Glória Dulce de Almeida Soares ${ }^{a *}$
}

\author{
${ }^{a}$ Department of Metallurgical and Materials Engineering, Federal University of Rio de Janeiro - UFRJ, \\ Av. Pedro Calmon, $n^{\circ}$ 550, CEP 21941-901, Rio de Janeiro, RJ, Brazil \\ ${ }^{b}$ Institute of Materials Science of Mulhouse, Haute-Alsace University, France
}

Received: September 14, 2011; Revised: November 23, 2011

\begin{abstract}
Hydroxyapatite-collagen (HA/Col) composites are potential scaffolds for bone tissue engineering. In this work, three-dimensional (3-D) HA/Col (50/50 wt. (\%)) scaffolds were synthesized using a selfassembly method and cross-linked with a $0.125 \%$ glutaraldehyde solution. Scaffolds were evaluated in vitro by cytotoxicity testing using MC3T3 cells; proliferation and differentiation were studied using STRO-1A human stromal cells for up to 21 days. Morphological and histological examinations showed a fibrous structure with a good distribution and homogeneous HA particles distribution. By thermogravimetric analysis, a ratio of 1.2 between inorganic and organic phase was found. The scaffolds presented no cytotoxicity when evaluated using three different parameters of cell survival and integrity: 2,3-bis[2-methyloxy-4-nitro-5-sulfophenyl]-2H-tetrazolium-5-carboxanilide (XTT), Neutral Red (NR) and Crystal Violet Dye Elution (CVDE). STRO-1A cells were found to adhere, proliferate and differentiate on the 3-D scaffold, but limited cell penetration was observed.
\end{abstract}

Keywords: biomaterials, tissues engineering, hydroxyapatite, collagen, cell culture, scaffolds

\section{Introduction}

Tissue engineering (TE) presents an attractive approach to regenerate damaged or diseased living tissue. The combination of living cells, biologically active molecules and a structural scaffold is the basis of TE technology. The most commonly applied strategy focuses on culturing cells in a temporary $3-\mathrm{D}$ matrix that mimics the natural extracellular matrix (ECM). Therefore, the chemical composition, controlled biodegradability and biologically functional properties are all important attributes for scaffolds used in $\mathrm{TE}^{1-3}$.

Calcium phosphates such as hydroxyapatite (HA; $\left.\mathrm{Ca}_{10}\left(\mathrm{PO}_{4}\right)_{6}(\mathrm{OH})_{2}\right)$ have shown good biocompatibility and osteoconductivity ${ }^{4,5}$, although their mechanical strength limits their use to non-load bearing applications ${ }^{6,7}$. HA synthesis by wet precipitation is largely used and can be obtained by different routes. On the other hand, natural polymers, like collagen $(\mathrm{Col})$ fibers, exhibit good compatibility and low immunogenicity. Composites based on $\mathrm{HA}$ and $\mathrm{Col}$ have been shown an increase of mechanical properties compared to Col-like materials ${ }^{6,7}$. Moreover, collagen-based scaffolds can be chemically modified by some reactions with reactive amine, carboxylic acid and hydroxyl groups. Those reactions allow the improvement of different structural, mechanical and physico-chemical properties $^{8}$.

The use of Col fibrils dissociated in a solution seems to provide an effective interaction between Col molecules and the mineral phase precipitated in comparison with other composite routes ${ }^{1,2,5,7-9}$. The cross-linking process induces a significant change in the $\mathrm{Col}$ structure from the nanoscale to microscale range $e^{8,10,11}$ and it is usually necessary in

*e-mail: gloria@metalmat.ufrj.br order to reduce its immunogenicity and control material biodegradation ${ }^{11}$. Biodegradation, local cytotoxicity and mechanical function may be explored to improve cell behavior.

HA-polymer composite scaffolds can improve osteoblastic cell growth. Moreover, they significantly enhance the expression of mature bone marker genes such as osteocalcin and bone sialoprotein ${ }^{12}$. Finally, HA/ Col scaffolds have shown important roles in TE, with good in vitro and in vivo results ${ }^{13-15}$, but few studies have analyzed the influence of the process parameters used for scaffold preparation on their in vitro biological functionality.

Several process parameters, such as raw materials, $\mathrm{pH}$, temperature, aging time and the presence of precursor substances, can affect the morphology and the mineral content of synthetic scaffolds ${ }^{16}$. Moreover, in the selfassembly of $\mathrm{HA} / \mathrm{Col}$, for example, chemical interactions between HA nanocrystals and polar groups, including carboxyl $(-\mathrm{COOH})$, amino $\left(-\mathrm{NH}_{2}\right)$ and hydroxyl $(-\mathrm{OH})$ groups, on $\mathrm{Col}$ fibers can be affected by collagen extraction.

When implanted in the body, the biomaterial undergoes many physiological processes which lead to several modifications in the biomaterial. The stability of the material is important after implantation, and the success of the graft depends on the capability of the material to remodel itself and to maintain cell colonization ${ }^{8,10,11}$. Investigations into the material's capacity for cell colonization should be assessed in a pre-surgical step, before implantation. Microporosity and biocompatibility play an important role in cell behavior when cells are cultured in three-dimensional scaffolds ${ }^{1-3}$.

The aim of the current study was to produce 3-D HA/ Col (50/50 wt. (\%)) composite scaffolds, to investigate their biocompatibility through a cytotoxicity test and, finally, to 
evaluate them in vitro in the presence of immortalized human stromal (STRO-1A) cells.

\section{Materials and Methods}

\subsection{Collagen extraction}

Isolation of Col fibrils from bovine Achilles tendon was performed by the enzymatic action of pepsin (SigmaAldrich, UK), which cleaves telopeptides. The bovine tendon was washed and cut into $3 \mathrm{~mm}^{3}$ cubes. The pieces were crushed in a food processor and added to a solution of $0.5 \mathrm{M}$ acetic acid (Merck, Brazil) and $10 \%$ pepsin, then incubated at $30{ }^{\circ} \mathrm{C}$ for 24 hours. The extraction solution was centrifuged at 90,000 g (Eppendorf, 5810R, Germany) for complete removal of impurities. The fibers were separated and precipitated with solution of $10 \% \mathrm{NaCl}$ (Vetec, Brazil). The precipitated fibers were dialyzed in distilled water for 3 days and redissociated in $59.32 \mathrm{mM}$ orthophosphoric acid (Merck, Brazil). The final fiber solution (at a concentration of $12 \mathrm{mg} \cdot \mathrm{mL}^{-1}$ ) was stored at $4{ }^{\circ} \mathrm{C}$ until use.

\subsection{Synthesis and cross-linking of $\mathrm{HA} / \mathrm{Col}$ composites}

The HA/Col (50:50 wt. (\%)) 3-D composite scaffolds were produced by the aqueous precipitation method in the presence of collagen fibers; this is also known as the self-assembly method ${ }^{11}$. The theoretical $\mathrm{Ca} / \mathrm{P}$ ratio and temperature was equal to 1.67 and $38{ }^{\circ} \mathrm{C}$, respectively, and the $\mathrm{pH}$ was maintained at $8-9^{7,9}$. For this, calcium nitrate solution (37.2 $\mathrm{mM}$ ) and Col fibers in orthophosphoric acid solution (59.32 mM) were gradually, and in parallel, added through peristaltic pumps into a reaction vessel with $25 \mathrm{~mL}$ of ultrapure water, followed by an aging time of 3 hours. Three syntheses were prepared and cylindrical molds ( $~ 8 \mathrm{~mm}$ diameter and $3 \mathrm{~mm}$ high) were filled with the produced materials and lyophilized.

The synthesized 3-D composites were kept in saline solution (PBS) for 24 hours before cross-linking with $0.125 \%$ glutaraldehyde (TedPella, USA) and sterilized with a total dose of $25 \mathrm{kGy}$ using $\gamma$ radiation. The glutaraldehyde concentration was previously determined, based on the literature ${ }^{10,17}$, considering that the scaffold had to maintain its integrity in culture medium for at least 28 days. By thermogravimetric analysis (results not shown) a ratio of 1.2 between organic and inorganic phase was found.

Fourier-transform infrared spectroscopy (FTIR) analysis of the composite, before and after cross-linking, was carried out using the Spectrum One FTIR (ABB Bomem Inc., USA) system. For this analysis, the scaffolds were finely cut and mixed with potassium bromide $(\mathrm{KBr})$. Spectra were collected in the wavenumber range of $4000-400 \mathrm{~cm}^{-1}$ at a nominal resolution of $4.00 \mathrm{~cm}^{-1}$ and with a number of scans equal to 100 .

\subsection{Morphological characterization}

The morphology of the cross-linked HA/Col scaffolds, before and after culture with STRO-1A cells, was observed by scanning electronic microscopy (SEM). The 3 -D scaffolds were fixed with glutaraldehyde (4\%) and paraformaldehyde $(2 \%)$ in phosphate buffered saline (PBS; pH 7.4). They were dehydrated in increasing ethanol solutions (50, 70, 80, 95 and 100\%) and immersed twice for 10 minutes in each solution. After complete dehydration using hexamethyldisilasane (HMDS; SigmaAldrich, UK), the scaffolds were gold-coated and examined under a Quanta 400 environmental scanning electron microscope (FEI, USA).

For transmission electron microscopy (TEM) analysis, the 3-D cross-linked HA/Col scaffold was embedded in Spurr ${ }^{\mathrm{TM}}$ and cut into thin sections. The transmission electron images were obtained on finely sliced samples using a Tecnai microscope (FEI, USA) with high resolution at $300 \mathrm{kV}$.

For fluorescence microscopy, an Olympus BX 51 microscope was utilized. The scaffolds were incubated for 1 hour in Bouin's solution (Sigma-Aldrich, UK) at room temperature. Next, samples were kept for 90 minutes in a siriusred solution $(0.1 \%$ saturated picric acid; SigmaAldrich, UK) and washed in $0.01 \mathrm{M}$ hydrochloric acid.

\subsection{Cytotoxicity test}

Samples were immersed in culture medium $(100 \mathrm{mg} /$ $\mathrm{mL}$ of cross-linked HA/Col scaffold in Dubelcco's Modified Eagle Medium (DMEM) free of fetal bovine serum) at $37{ }^{\circ} \mathrm{C}$ for 24 hours; the extracts were collected for the cytotoxicity assay according to ISO 10993-12 18 and $10993-5^{19}$. A $1 \%$ phenol solution was used as the positive cytotoxicity control and titanium powder $(100 \mathrm{mg} / \mathrm{mL})$ as the negative cytotoxicity control. Then, MC3T3-E1 osteoblasts (subclone 14-CRL 2594; ATCC) were seeded in a 96-well cell culture plate $\left(1 \times 10^{4}\right.$ cell/well $)$ and cultured in DMEM containing $\mathrm{NaHCO}_{3}\left(1.2\right.$ g.L $\left.\mathrm{L}^{-1}\right)$, ampicillin (0.025 g.L $\left.{ }^{-1}\right)$ and streptomycin $\left(0.1\right.$ g.L. $\left.\mathrm{L}^{-1}\right)$ supplemented with $10 \%$ fetal bovine serum for 24 hours at $37^{\circ} \mathrm{C}$ in $5 \% \mathrm{CO}_{2} / 95 \%$ air.

After 24 hours of cell exposure to the extract media, cytotoxicity was evaluated with a commercial kit (Cytotox, Xenometrix, Germany) which allows the use of three different parameters of cell survival and integrity on the same sample: 2,3-bis[2-methyloxy-4-nitro-5sulfophenyl]-2H-tetrazolium-5-carboxanilide (XTT), Neutral Red (NR) and Crystal Violet Dye Elution (CVDE). The absorbance data were obtained using a microplate UV/Vis spectrophotometer (PowerWave MS2, BioTek Instruments, USA).

\subsection{Cell culture with osteoprogenitor stromal cells}

Immortalized human stromal cells (STRO-1A) were graciously provided by Marie et al. ${ }^{20}$. When STRO-1A cells had reached confluence, the cells were harvested, detached with trypsin-ethylenediamine tetra-acetic acid (EDTA; Sigma-Aldrich, UK), counted $\left(5 \times 10^{5}\right.$ cells/scaffold $)$ and resuspended in culture medium (Iscove's medium with L-glutamine containing $10 \% \mathrm{SBF}, 100 \mathrm{U} / \mathrm{mL}$ penicillin $\mathrm{G}$, $100 \mu \mathrm{g} / \mathrm{mL}$ streptomycin sulfate and $10^{-8} \mathrm{M}$ dexamethasone; Sigma-Aldrich, UK). The sterilized scaffolds were rehydrated with complete cell culture medium for 24 hours before cell culture. After this period, STRO-1A cells were inoculated into the scaffolds and cultured under static conditions for 24 hours, 3, 7, 14 and 21 days in a humid 


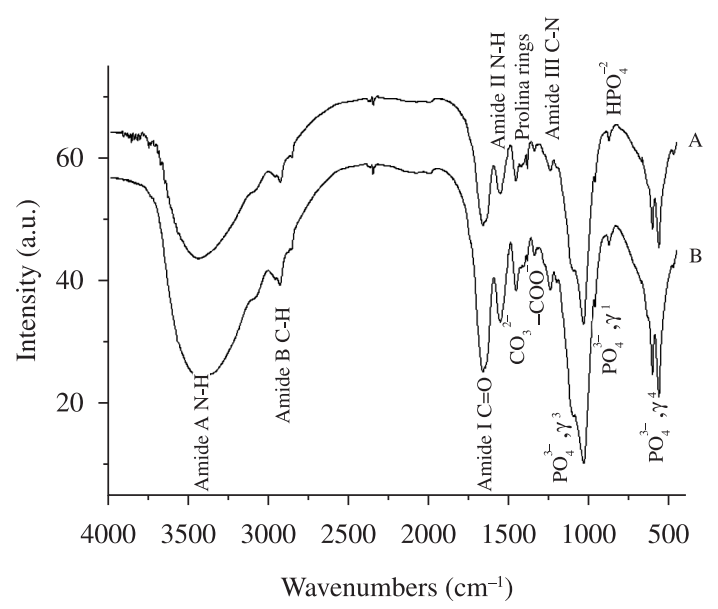

Figure 1. FTIR spectra of the HA/Col (50/50 wt. (\%)) composite before (A) and after cross-linking with $0.125 \%$ glutaraldehyde (B). incubator at $37{ }^{\circ} \mathrm{C}$ and $5 \% \mathrm{CO}_{2}$. The medium was renewed three times a week.

The proliferation of STRO-1A cells on crosslinked HA/Col scaffolds was determined using the MTT (3-\{4,5-dimethylthiazol-2-yl $\}-2,5$-diphenyl-2Htetrazolium-bromide; Sigma-Aldrich, UK) assay. The samples $(n=6)$ were incubated in $500 \mu \mathrm{L}$ of MTT solution at $37{ }^{\circ} \mathrm{C}$ in a humid atmosphere containing $5 \% \mathrm{CO}_{2}$ for 3 hours. After the complete withdrawal of MTT, $500 \mu \mathrm{L}$ of acidic isopropanol $(0.3 \%)$ were added to the samples. After 10 minutes, the optical density was read at $570 \mathrm{~nm}$ on a microplate reader (ELX, 800UV, Biotec Instruments, INC).

Alkaline phosphatase (ALP) activity was measured using p-nitrophenylphosphate as the substrate in an alkaline buffer solution $(20 \mathrm{mM}$ p-nitrophosphate $+100 \mathrm{mM}$ diethanolamine $98 \%+10 \mathrm{mM} \mathrm{MgCl}_{2}, \mathrm{pH} 9.5$ at room temperature). The scaffolds $(n=6)$ were permeabilized with a $0.5 \%$ aqueous solution of Triton X-100 (Sigma-Aldrich,

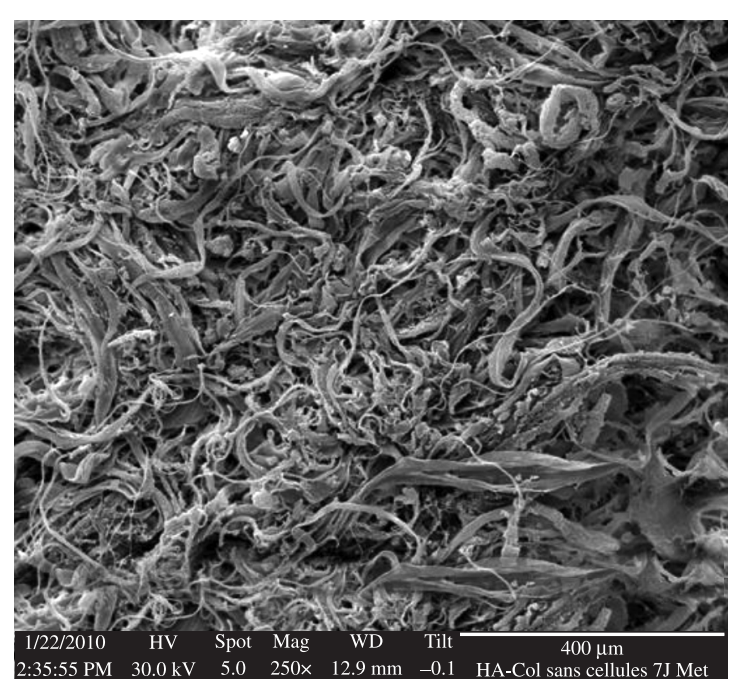

(a)

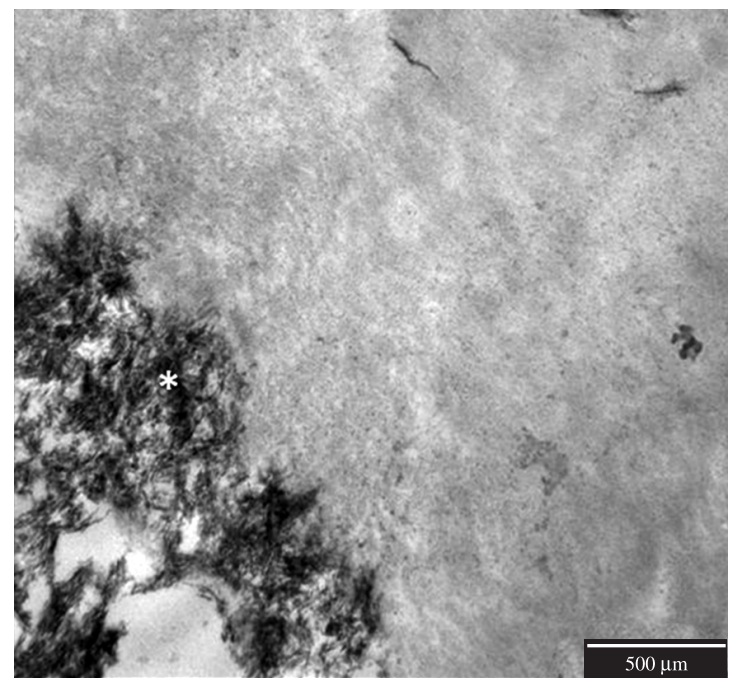

(c)

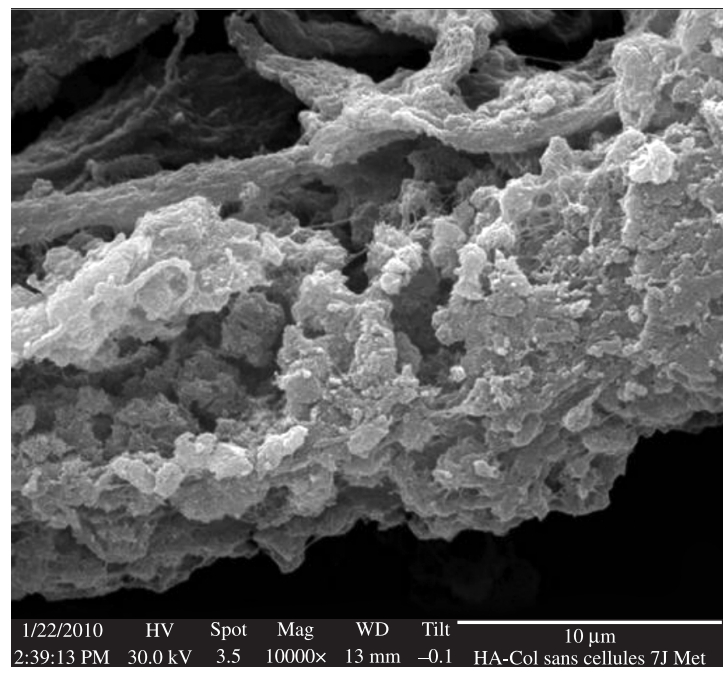

(b)

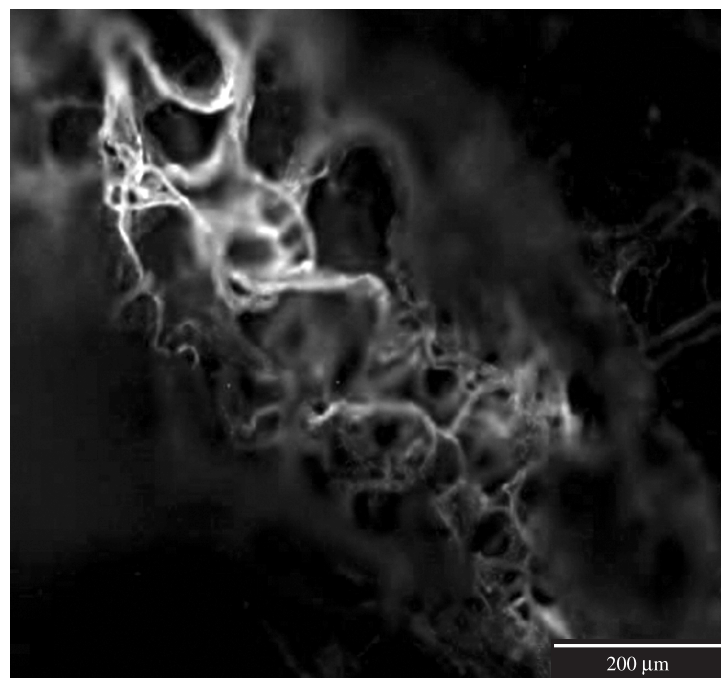

(d)

Figure 2. SEM micrographs of the HA/Col scaffold surface (a), detail of collagen fiber mineralization (b), TEM micrograph of the HA/Col scaffold showing deposits of HA crystals (*) around Col fibers (c) and picrosirius red staining for collagen identification (d). 
UK) and incubated for 30 minutes at $37^{\circ} \mathrm{C}$. Then, the reaction with the substrate was terminated with the addition of an EDTA solution (0.1 M EDTA in $1 \mathrm{M} \mathrm{NaOH})$. The optical density of the solution was read on a plate reader at $405 \mathrm{~nm}$. The osteocalcin (OC) and pro-collagen I (PIP) analyses were carried out according to the manufacturer's data using the Gla-Type Osteocalcin and Procollagen Type I C-Peptide EIA Kits, respectively (Takara Bio INC, Japan). After cell culture for 7, 14 and 21 days, aliquots of culture medium were incubated on one pre-treated 96-well plate at $37^{\circ} \mathrm{C}$. The optical density of the solution was read on a plate reader at $450 \mathrm{~nm}$.

The scaffolds cultured with STRO-1A cells for 24 hours and 14 days were also prepared for histological observation $(n=4)$. For this, the scaffolds were fixed with neutral-buffered formalin solution (10\%; Sigma-Aldrich, UK). Standard dehydration in sequentially increasing ethanol solutions to $100 \%$ ethanol was performed, followed by immersion in xylene (Sigma-Aldrich, UK), paraffin saturated xylene and finally molten paraffin (Fisher Scientific, UK). Blocks were sectioned at $15 \mu \mathrm{m}$ and stained

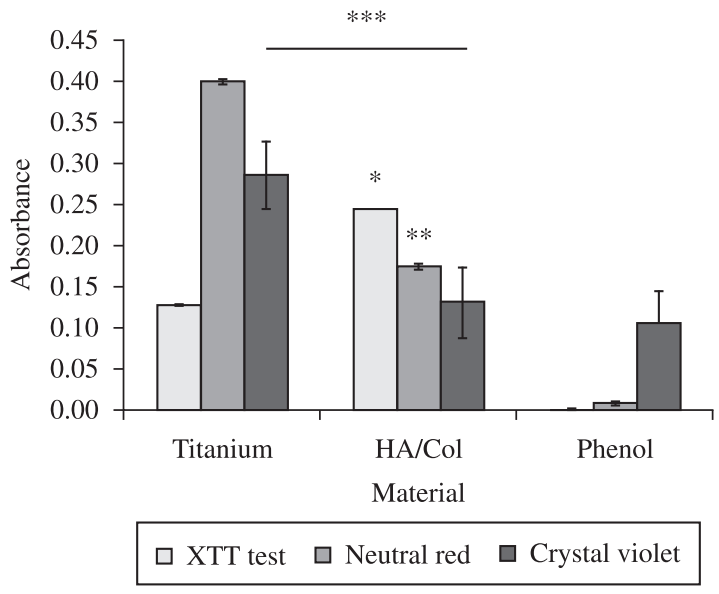

Figure 3. Cytotoxicity assay (XTT reduction, Neutral red uptake, and Crystal violet dye elution) of cross-linked HA/Col scaffold using MC3T3 cells. For XTT and NR test, statistically significant differences between groups were observed $(\mathrm{p}<0.05)(*, * *)$. For Crystal violet test $(* * *)$ denotes significant difference between HA/Col sample and titanium, $\mathrm{p}<0.05$. with 4',6-diamidino-2-phenylindole dihydrochloride (DAPI; Sigma-Aldrich, UK) for the visualization of cell nuclei.

\subsection{Statistical analysis}

Quantitative data are presented as mean \pm standard deviation (SD). After the Shapiro-Wilk normality test, ANOVA (one-way) and Tukey post-hoc tests were performed to determine the statistical significance between experimental groups. A value of $\mathrm{p}<0.05$ was considered to be statistically significant.

\section{Results and Discussion}

Recent studies have described different methods for producing HA/Col scaffolds, among which different selfassembly methods can be highlighted ${ }^{7-9,15,21,22}$. The samples obtained by the methods described above were visually homogeneous, with a three-dimensional network and were easy to manipulate. Moreover, samples could be produced in any size or shape.

The FTIR spectra, before and after cross-linking, for the HA/Col scaffold were similar and can be seen in Figure 1. Bands related to the phosphate characteristics were observed at $473 \mathrm{~cm}^{-1}$ (v2 mode), 560 and $604 \mathrm{~cm}^{-1}\left(\mathrm{PO}_{4}{ }^{3-}, \mathrm{v} 4\right.$ mode), $961 \mathrm{~cm}^{-1}\left(\mathrm{PO}_{4}^{3-}, \mathrm{v} 1 \mathrm{mode}\right)$ and $1030 \mathrm{~cm}^{-1}\left(\mathrm{PO}_{4}^{3-}, \mathrm{v} 3\right.$ mode $)$. After the cross-linking process, we observed only a moderate increase in the II and III amide bands similar to that observed by Chang and Tanaka ${ }^{23}$ and Wopenka et al. ${ }^{24}$.

The morphology of the HA/Col scaffold cross-linked with glutaraldehyde showed a relatively dense fibrous structure (Figure 2a). At higher magnification, the collagen fibers appeared to be covered by a calcium-phosphate layer (Figure 2b) which was confirmed by TEM examination. In Figure 2c, using TEM micrography, the mineral phase appeared as deposits covering the Col fibers. The mineral phase was rich in calcium and phosphorus elements (determined by energy-dispersive spectroscopy, not shown) and therefore called HA. Moreover, these needlelike HA crystals showed an irregular crystal orientation. However, the collagen was still accessible at some portion of the fiber surface as confirmed by picrosirius red staining, a specific collagen marker (Figure $2 \mathrm{~d}$ ).

These results are in accordance with those of Cool et al. ${ }^{25}$ who demonstrated that only $25 \%$ of the mineral phase was

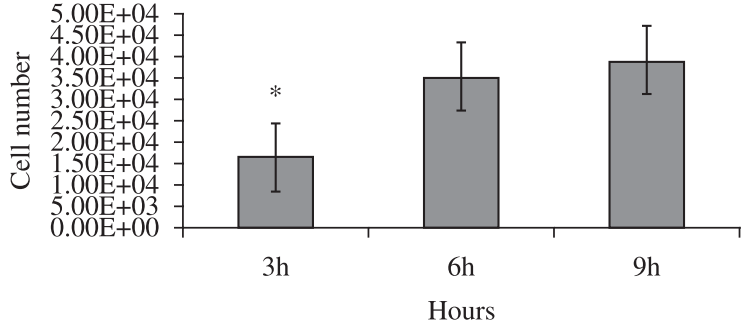

(a)

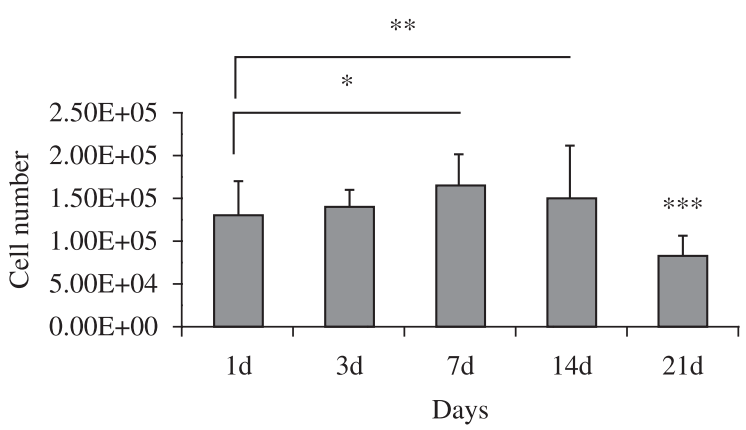

(b)

Figure 4. MTT assay of STRO-1A cells at several incubation periods (A, hours and B, days) on cross-linked HA/Col scaffold. Error bars represent means $+/-\mathrm{SD}$ for $\mathrm{n}=6$. The symbols $(* * *, * * *)$ indicate statistical significance within a time point $(\mathrm{p}<0.05)$. 
deposited inside fibril gaps, while the remaining $75 \%$ of the precipitates were found on the Col fiber surface without any specific orientation. Several different factors seem to act, synergistically or interchangeably, to initiate mineral deposition in organic structures. Zhang et al. ${ }^{15}$ suggested that mineral nucleation and growth is mediated by a combination of biological, physico-chemical and biomechanical factors. The presence or absence of proteins and other substances, such as proteoglycans, can influence the nucleation phase. In our case, during HA/Col scaffold preparation, Col fibers quickly agglomerated together with the HA nanocrystals. Finally, organic clusters with entrapped HA crystals were formed, limiting crystal growth due to their confinement among the Col fibers.

Figure 3 shows the in vitro cytotoxicity results evaluated by XTT, NR and CVDE after 24 hours in culture on the cross-linked HA/Col scaffold extracts. The XTT results showed a statistically superior metabolic activity on HA/ Col scaffolds compared to the titanium sample (negative cytotoxicity control). Regarding the NR assays, there was a statistically significant difference between the titanium sample and the HA/Col scaffolds, with the latter presenting poorer cell membrane integrity; however, this parameter was significantly greater than in the phenol sample (positive cytotoxicity control). Similarly, the crystal violet assays showed a lower cell number with HA/Col scaffold extracts compared to titanium material extracts. Considering these assays, the optimized cross-linked HA/Col scaffold was considered to be cytocompatible and tests with human stromal cells were initiated ${ }^{26}$.

The possibility that the HA/Col scaffolds could be used as a carrier for culturing human stromal cells was

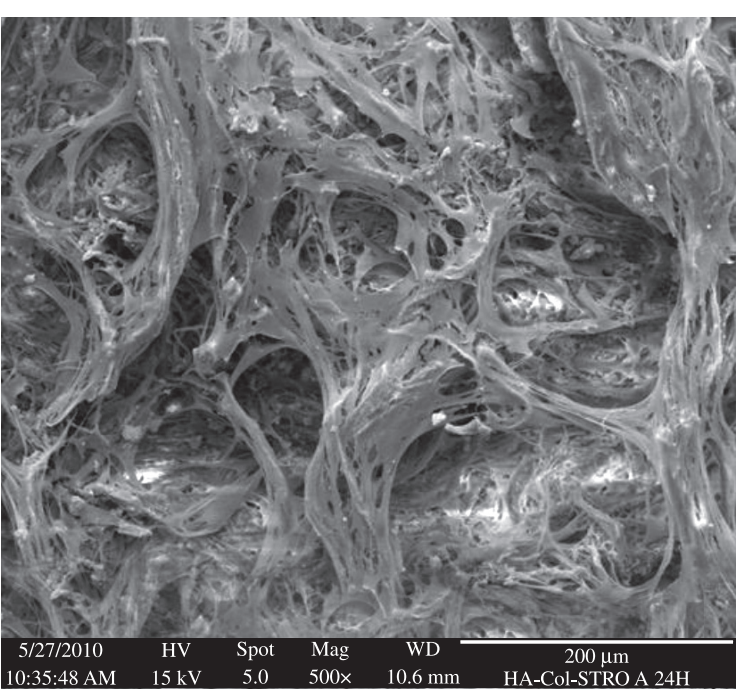

(a)

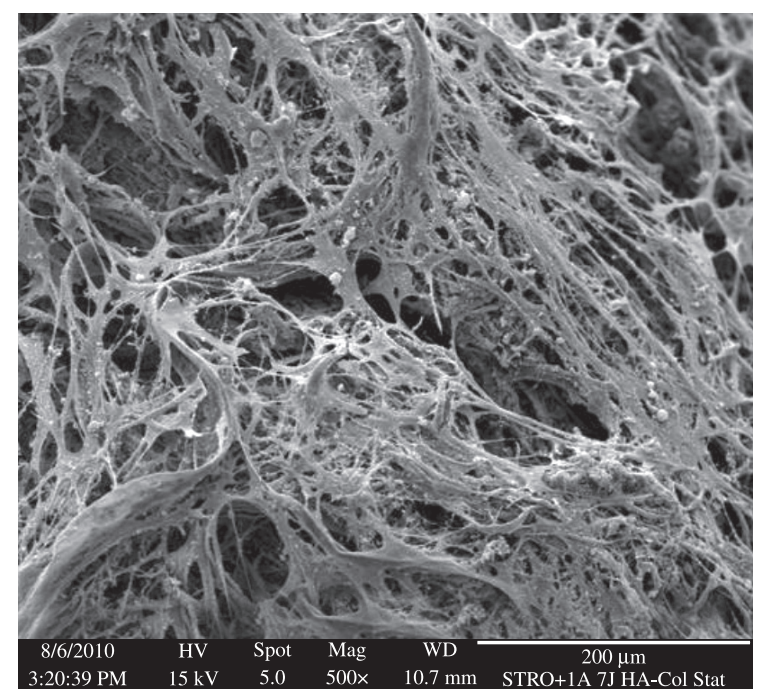

(c)

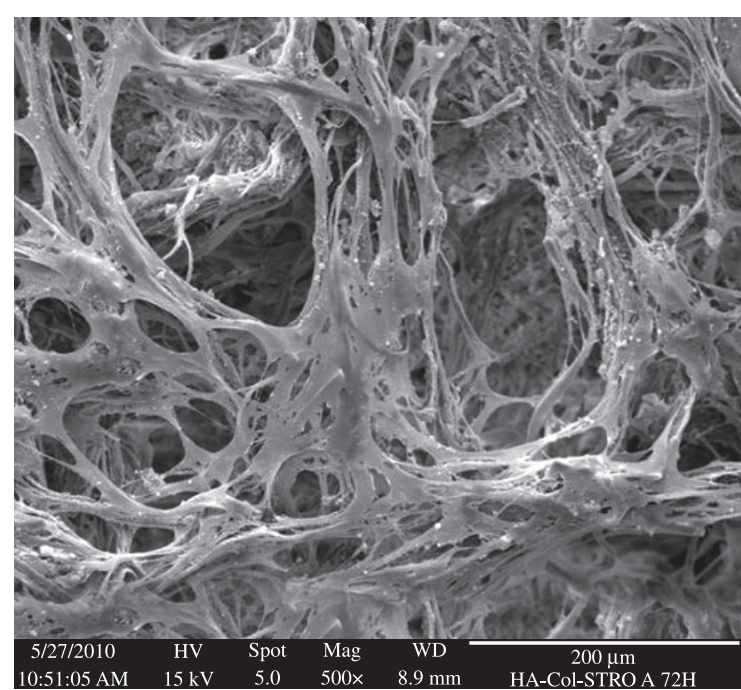

(b)

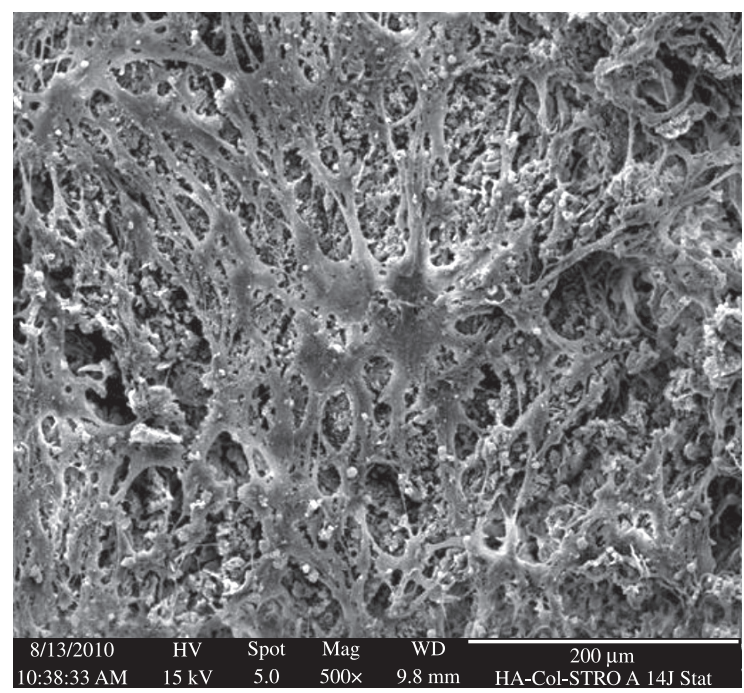

(d)

Figure 5. SEM micrographs of STRO-1A cells cultured on cross-linked 3-D HA/Col scaffolds for: a) 24 hours; b) 3; c) 7; and d) 14 days. Original magnification: $500 \times$. 
examined. For this, STRO-1A cells were cultured for up to 21 days on the scaffolds, and their ability to adhere, colonize and proliferate inside the scaffold was examined. The STRO-1A cells needed 6 hours to completely attach to the scaffolds (Figure 4a). Furthermore, they started to proliferate. The highest number of cells on the scaffolds was observed after 7 days. However, after this time, the number of cells decreased, and after 21 days, half of the cells had died, probably because of the culture conditions used (Figure 4b). Indeed, some authors have shown that to maintain viable cells inside 3-D scaffolds for long periods, it is necessary to force medium perfusion inside the scaffold using dynamic culture conditions ${ }^{27-29}$.

Regarding cell morphology on scaffolds, after the first 24 hours, STRO-1A cells already showed adhesion and spreading processes, demonstrating the biocompatibility of the cross-linked HA/Col 3-D scaffold (Figure 5a). Due to the porous surface and irregular topography, cells exhibited a polygonal shape and many filopodial structures forming network interactions between cell membranes and mineralized collagen fibers, as can be observed in Figures $5 \mathrm{~b}$ and 5c. After 14 days, the surface of the HA/Col scaffold was almost covered with cells and possibly mixed with secreted ECM (Figure 5d). Indeed, it has been previously demonstrated that STRO-1A cells are capable of secreting and mineralizing an ECM for up to 21 days in culture, when stimulated by dexamethasone and $1,25(\mathrm{OH})_{2} \mathrm{D}_{3}{ }^{20}$. Thus, from this morphological study, it appears that the HA/Col scaffold can be a favorable microenvironment for the growth of STRO-1A cells during two weeks of culture.
Figure 6 shows the differentiation state of STRO-1A cells after 7, 14 and 21 days of culture on HA/Col scaffolds. Alkaline phosphatase activity (ALP, Figure 6a) showed a regular increase over time with a significantly higher value after 21 days. A second primary differentiation marker, the type I collagen synthesis (PIP, Figure 6c), showed a similar trend. On the contrary, the late differentiation marker, osteocalcin (OC, Figure 6b), showed a significantly higher value only after 21 days. Normally, the alkaline phosphatase activity and type I collagen synthesis should be higher at earlier times and osteocalcin synthesis at later times ${ }^{12,20}$. This discrepancy could be explained by decreased cell viability after 14 days.

The nuclei of STRO-1A cells were observed after DAPI staining on histological sections of the HA/ Col scaffold after 24 hours and 14 days in order to evaluate the capacity of cells to penetrate into the scaffold. The image in Figure 7a shows that cells had already migrated into the HA/Col scaffold after 24 hours. However, after 14 days, as shown in Figure 7b, proliferation was greater outside the scaffold and a continuous cell layer was formed on the surface, as also observed on the SEM image (Figure 5d). The number of cells inside the scaffold did not increase over time. This suggests that static conditions are not sufficient to allow the survival of cells inside the scaffold and that dynamic culture conditions with perfusion of the scaffold by the culture medium must be established. These studies are currently in progress.

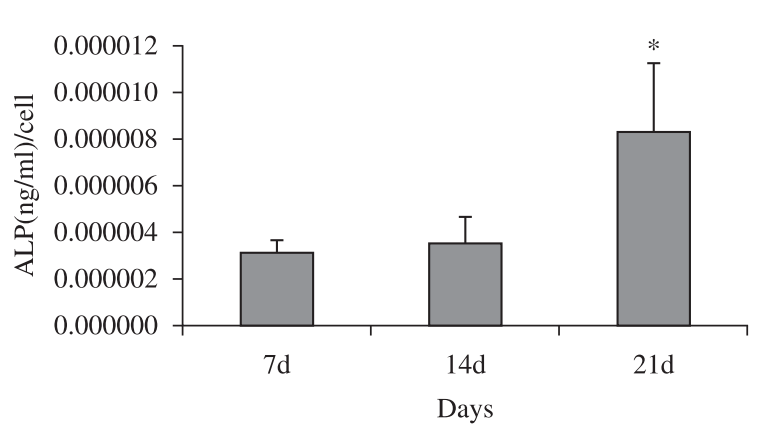

(a)

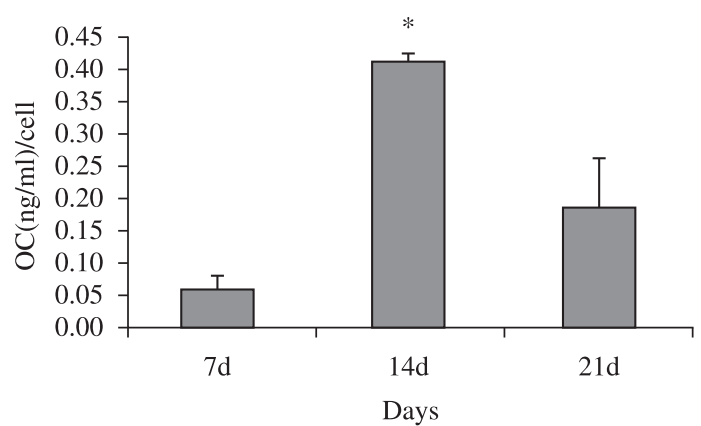

(b)

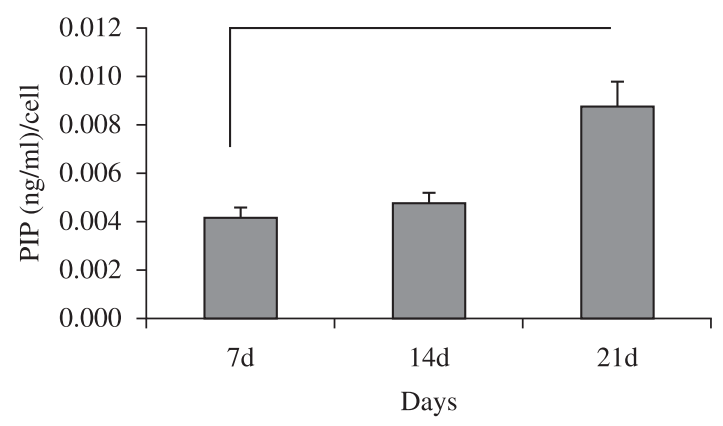

(c)

Figure 6. Differentiation expression of STRO+1A cells cultured on cross-linked HA/Col scaffolds for up to 21 days. Alkaline phosphatase (a), osteocalcin (b) and pro-collagen I (c)-PIP expression. Error bars represent mean \pm SD with $n=6$. The symbol (*) indicates statistical significance within a time point $(\mathrm{p}<0.05)$. 


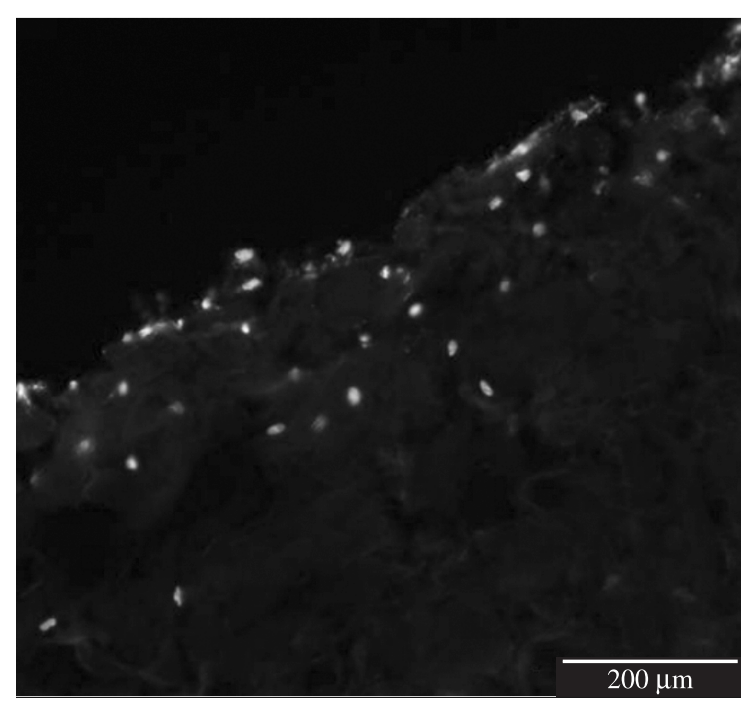

(a)

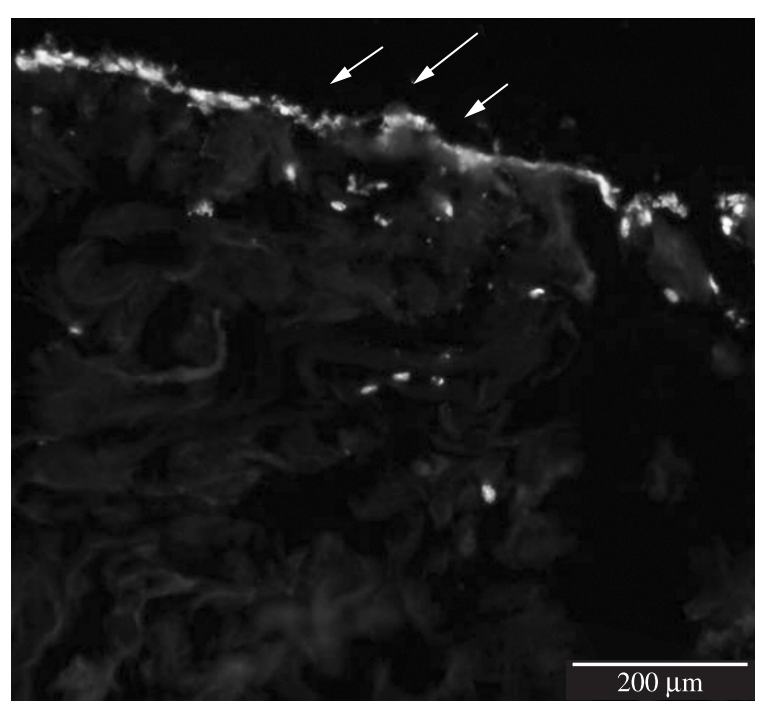

(b)

Figure 7. DAPI staining of STRO-1A cells cultured on crosslinked HA/Col scaffold cross-sections for: a) 24 hours; and b) 14 days. Details of continuous cell layer formed on the surface (b).

\section{Conclusion}

In this work, 3-D HA/Col (50/50 wt. (\%)) scaffolds were synthesized using a self-assembly method and crosslinked with $0.125 \%$ glutaraldehyde. The scaffolds presented no cytotoxicity as evaluated using MC3T3 cells by three different parameters of cell survival and integrity on the same sample: 2,3-bis[2-methyloxy-4-nitro-5-sulfophenyl]2H-tetrazolium-5-carboxanilide (XTT), Neutral Red (NR) and Crystal Violet Dye Elution (CVDE). STRO-1A cells were found to adhere, proliferate and differentiate on the
3-D scaffold, but limited cell penetration inside the scaffolds was observed.

\section{Acknowledgements}

The authors would like to thank FAPERJ, CNPq and the CAPES-COFECUB project $n^{\circ} 628 / 09$ for financial support. The authors also acknowledge the Biomineralization Laboratory/ICB/UFRJ for TEM analysis and the Bioengineering, Biomaterials and Biological Mineralization Laboratory/UPC-HUAP/UFF for the cytotoxicity tests.

\section{References}

1. Hutmacher DW, Chantz JT, Lam CXF, Tan KC and Lim TC. State of the art and future directions of scaffold-based bone engineering from a biomaterials perspective.Journal of Tissue Engineering and Regenerative Medicine.2007; 1:245-260. PMid:18038415. http://dx.doi.org/10.1002/term.24

2. Ma PX. Biomimetic materials for tissue engineering.Advanced Drug Delivery Reviews.2008; 60:184-198.PMid:18045729. PMCid:2271038. http://dx.doi.org/10.1016/j.addr.2007.08.041

3. Rosso F, Marino G, Giordano A, Barbarisi M, Parmeggiani D andBarbarisi A. Smart materials as scaffolds for tissue engineering. Journal of Cellular Physiology.2005; 203:465-470. PMid:15744740. http://dx.doi.org/10.1002/jcp.20270

4. Saldana L, Sanchez-Salcedo S, Izquierdo-Barba I, Bensiamar F, Munuera L, Vallet-Regı M et al. Calcium phosphate-based particles influence osteogenic maturation of human mesenchymal stem cells. ActaBiomaterialia.2009; 5:1294-1305.PMid:19114315. http://dx.doi.org/10.1016/j. actbio.2008.11.022

5. Tachibana A, Kaneko S, Tanabe T and Yamauchi K. Rapid fabrication of keratin-hydroxyapatite hybrid sponges toward osteoblast cultivation and differentiation. Biomaterials.2005; 26:297-302.PMid:15262471. http://dx.doi.org/10.1016/j. biomaterials.2004.02.032

6. Ouahi M, Champion E, Gallet O, Jada A andAnselme K. Physico-chemical characteristics and protein adsorption potential of hydroxyapatite particles: influence on in vitro biocompatibility of ceramics after sintering. Colloids And Surfaces B: Biointerfaces.2006; 47:10-19.PMid:16387480. http://dx.doi.org/10.1016/j.colsurfb.2005.11.015

7. Kikuchi M, Itoh S, Ichinose S, Shinomiya K and Tanaka J. Self-organization mechanism in a bone-like hydroxyapatite/ collagen nanocomposite synthesizes in vitro and its biological reaction in vivo. Biomaterials.2001; 22:17051711. http://dx.doi.org/10.1016/S0142-9612(00)00305-7

8. Wahl DA andCzernuszka JT. Collagen-hydroxyapatite composites for hard tissue repair. European Cells and Materials.2006; 11:43-56.PMid:16568401.

9. Cunniffe GM, Dickson GR, Partap S, Stanton KT and O'Brien FJ. Development and characterisation of a collagen nanohydroxyapatite composite scaffold for bone tissue engineering. Journal of Materials Science: Materials in Medicine.2010; 21:2293-2298.PMid:20091099. http://dx.doi.org/10.1007/ s10856-009-3964-1

10. Charulatha V andRajaram A. Influence of different crosslinking treatments on the physical properties of collagen 
membranes. Biomaterials.2003; 24:759-767. http://dx.doi. org/10.1016/S0142-9612(02)00412-X

11. Kikuchi M, Matsumoto HN, Yamada T, Koyama Y, Takakuda $\mathrm{K}$ and Tanaka J. Glutaraldehyde cross-linked hydroxyapatite/ collagen self. organization nanocomposites. Biomaterials.2004; 25:63-69. http://dx.doi.org/10.1016/S0142-9612(03)00472-1

12. Kim K, Dean D, Lu A, Mikos AG and Fisher JP. Early osteogenic signal expression of rat bone marrow stromal cells is influenced by both hydroxyapatite nanoparticle content and initial cell seeding density in biodegradable nanocomposite scaffolds. ActaBiomaterialia.2011; 7:1249-1264.PMid:21074640. http://dx.doi.org/10.1016/j.actbio.2010.11.007

13. Itoh S, Kikuchi M, Koyama Y, Takakuda K, Shinomiya K and Tanaka J. Development of an artificial vertebral body using a novel biomaterial, hydroxyapatite/collagen composite. Biomaterials.2002; 23:3919-3926. http://dx.doi.org/10.1016/ S0142-9612(02)00126-6

14. Tcacencu IandWendel M. Collagen-hydroxyapatite composite enhances regeneration of calvaria bone defects in young rats but postpones the regeneration of calvaria bone in aged rats. Journal of Materials Science: Materials in Medicine.2008; 19:2015-2021.PMid:17952564. http://dx.doi.org/10.1007/ s10856-007-3284-2

15. Zhang L, Tang P, Xu M, Zhang W, Chai W and Wang Y. Effects of crystalline phase on the biological properties of collagen-hydroxyapatite composites. ActaBiomaterialia.2010; 6:2189-2199.PMid:20040387. http://dx.doi.org/10.1016/j. actbio.2009.12.042

16. Ficai A, Andronescu E, Voicu G, Ghitulica C andFicai D. The influence of collagen support and ionic species on the morphology of collagen/hydroxyapatite composite materials. Materials Characterization.2010; 61:402-407. http://dx.doi. org/10.1016/j.matchar.2010.01.003

17. Bigi A, Cojazzi G, Panzavolta S, Rubini K andRoveri N. Mechanical and thermal properties of gelatin films at different degrees of glutaraldehyde cross-linking. Biomaterials.2001; 22:763-768. http://dx.doi.org/10.1016/S0142-9612(00)00236-2

18. International Organization for Standardization - ISO.ISO 10993-5: Biological evaluation of medical devices. Geneva: ISO; 1999. Part 5: Tests for cytotoxicity: In vitro methods.

19. International Organization for Standardization . ISO. ISO 10993-12: Biological evaluation of medical devices. Geneva: ISO; 1996. Part 12: Sample preparation and reference materials.

20. Oyajobi BO, Lomri A, Hott M and Marie PJ. Isolation and characterization of human clonogenic osteoblast progenitorsimmunoselected from fetal bone marrow stroma using STRO-1 monoclonal antibody. Journal of Bone and Mineral Research. 1999; 14(3):351-361.PMid:10027900. http://dx.doi.org/10.1359/jbmr.1999.14.3.351

21. Sena LA, Caraballo MM, Rossi AM and Soares GA. Synthesis and characterization of biocomposites with different hydroxyapatite-collagen rations. Journal of Materials Science: Materials in Medicine.2009; 20:2395-2400.PMid:19585226. http://dx.doi.org/10.1007/s10856-009-3813-2

22. Gelinsky M, Welzel PB, Simon P, Bernhardt A andKonig U. Porous three-dimensional scaffolds made of mineralised collagen: preparation and proporties of a biomimetic nanocomposite material for tissue engineering of bone. Chemical Engineering Journal.2008; 137:84-96. http://dx.doi. org/10.1016/j.cej.2007.09.029

23. Chang MC and Tanaka J. FT-IR study for hydroxyapatite/ collagen nanocomposite cross-linked by glutaraldehyde. Biomaterials 2002; 23:4811-4818. http://dx.doi.org/10.1016/ S0142-9612(02)00232-6

24. Wopenka B andPasteris JD. A mineralogical perspective on the apatite in bone.Materials Science and Engineering.2005; 25:131-143. http://dx.doi.org/10.1016/j.msec.2005.01.008

25. Cool SM, Forwood MR, Campbell Pand Bennett MB. Comparisons between bone and cementum compositions and the possible basis for their layered appearances.Bone.2002; 30:382-392. http://dx.doi.org/10.1016/S8756-3282(01)00686-X

26. De Deus G, Ganabarro A andAlaves G. Optimal cytocompatibility of a bioceramicnanoparticulate cement in primary human mesenchymal cells. Journal of endodotics. 2009; 35: 1387-1390.

27. Yeatts $A B$ and Fisher JP. Tubular perfusion system for the longterm dynamic culture of human mesenchymal stem cells. Tissue Engineering: Part C. 2011; 17(3):337-348.PMid:20929287. http://dx.doi.org/10.1089/ten.tec.2010.0172

28. David B, Bonnefont-Rousselot D, Oudina K, Degat MC, Deschepper M, Viateau V et al. A perfusion bioreactor for engineering bone constructs: an in vitro and in vivo study. Tissue Engineering: Part C. 2011; 17(5):505-516. PMid:21171934. http://dx.doi.org/10.1089/ten.tec.2010.0468

29. McCoy RJ and O'Brien FJ. Influence of shear stress in perfusion bioreactor cultures for the development of three-dimensional bone tissue constructs: a review. Tissue Engineering:Part B. 2010; 16(6):587-601.PMid:20799909. http://dx.doi.org/10.1089/ten.teb.2010.0370 